\title{
Impact of Renal Insufficiency on Prognosis of Patients with Acute Coronary Syndrome
}

\author{
Lingyao Qi \\ Hanxiong Liu \\ Lianchao Cheng \\ Caiyan Cui \\ Xu Chen \\ Siqi Yang \\ Lin Cai (D)
}

Department of Cardiology, Affiliated Hospital of Southwest Jiaotong

University, The Third People's Hospital of

Chengdu, Chengdu, Sichuan, People's

Republic of China
Correspondence: Lin Cai

Department of Cardiology, Affiliated Hospital of Southwest Jiaotong University,

The Third People's Hospital of Chengdu, Chengdu, Sichuan, People's Republic of

China

Tel/Fax +86 28-67575956

Email clin63@hotmail.com
Purpose: Chronic kidney disease (CKD) is common in patients admitted with acute coronary syndrome (ACS), and it is associated with poor outcomes. However, data are limited. Hence, we examined the long-term prognostic significance of estimated glomerular filtration rate (eGFR) among Chinese patients hospitalized with ACS.

Patients and Methods: This is a multicenter, observational study that included 1860 ACS patients enrolled between March 2014 and June 2019 from 11 hospitals in Chengdu. CKDEPI equation was used to calculate the baseline eGFR. Patients were divided into three groups: eGFR $\geq 90 \mathrm{~mL} / \mathrm{min}$ (normal renal function), eGFR 60 to $<90 \mathrm{~mL} / \mathrm{min}$ (mild impaired renal function), and eGFR $<60 \mathrm{~mL} / \mathrm{min}$ (moderate or severe renal dysfunction). The endpoint was all-cause death during follow-up.

Results: At baseline, 714 patients had normal renal function, while 746 patients had mild impaired renal function, and 400 patients had moderate or severe renal dysfunction. In the follow-up of 15 months (10 months, 22 months), 261 (14.0\%) patients died;, 139 (34.8\%) in the moderate or severe renal dysfunction group, 94 (12.6\%) in the mild impaired renal function group, and $28(3.9 \%)$ in the normal renal function group (log-rank p-value from Kaplan-Meier analysis $<0.001$ ). In multivariable Cox Proportional hazard analysis, age, systolic blood pressure (SBP), heart rate, eGFR, ST-elevation myocardial infarction (STEMI), and percutaneous coronary intervention (PCI) were independent predictors of all-cause death.

Conclusion: In this study, among Chinese patients with ACS, renal insufficiency was associated with unfavorable long-term prognosis. Age, SBP, heart rate, eGFR, STEMI, and PCI could identify those at risk.

Keywords: renal insufficiency, acute coronary syndrome, long-term prognosis, retrospective study

\section{Introduction}

Acute coronary syndrome (ACS) is one of the leading causes of cardiovascular disease (CVD) deaths. ${ }^{1}$ Despite advances in management, the morbidity and mortality of ACS still remain high. According to the recent American Heart Association's Heart Disease and Stroke Statistics Update, ${ }^{2}$ the number of inpatients diagnosed with ACS in the United States reached 1,045,000 in 2016. And the data on 2363 hospitals in 2018 from the Centers for Medicare \& Medicaid Services Hospital Inpatient Quality Reporting Program has shown that the average 30-day mortality after acute myocardial infarction (AMI) was $13.6 \%$, with higher mortality observed in rural hospitals (from $13.4 \%$ to $13.8 \%$ for the most urban to most rural hospitals). A nationwide study of Danish in 2020 showed the crude cumulative 1 -year incidence of all-cause mortality was $15.9 \%$ for patients 
with ACS. ${ }^{3}$ Meanwhile, the morbidity of ACS keeps rising in China, along with the increasing overall mortality of AMI from 2002 to 2018 . $^{4}$

Chronic kidney disease (CKD) is one of the major risk factors for CVD. The Global Burden of Disease, Injuries, and Risk Factors Study showed that the global all-age prevalence of CKD has increased by $29.3 \%$ from 1990 to $2017 .^{5}$ In 2012, a cross-sectional survey showed the overall prevalence of CKD was $10.8 \%$ in China; therefore, the number of patients with CKD is estimated to be about 119.5 million. ${ }^{6}$ Additionally, previous study showed that up to $29.0 \%$ of ACS patients are complicated with chronic kidney disease (CKD). ${ }^{7}$ On the other hand, renal dysfunction is one of the main risk factors for adverse outcomes in ACS. $^{8-10}$ Similarly, CVD is a leading cause of death in CKD patients. ${ }^{11,12}$ The two diseases are tightly linked and progression of either leads to worse of the other in a vicious cycle.

However, a few studies examined the long-term prognosis among Chinese ACS patients with renal insufficiency. And the prevalence of CKD was high southwest (18.3\% [16.4-20.4]) region compared with other regions. ${ }^{6}$ Therefore, this study sought to investigate the impact of renal insufficiency - assessed by estimated glomerular filtration rate (eGFR) - on admission of the long-term prognosis of ACS patients in the southwestern region of China.

\section{Patients and Methods Study Design and Population}

This is a multicenter, observational cohort study. We observed 1860 consecutive ACS patients admitted to 11 general hospitals between January 2014 and June 2019 in Chengdu, China (http://www.medresman.org). The study is registered in the Chinese Clinical Trials Registry in China (ChiCTR1900025138). ACS was defined according to the current guidelines. ${ }^{13,14}$ The ACS definition included ST-segment elevation myocardial infarction (STEMI) and non-ST-segment elevation myocardial infarction (NSTEMI), and unstable angina (UA). NSTEMI and UA are referred to as non-ST-segment elevation acute coronary syndrome (NSTE-ACS). We excluded the following patients: 1) patients younger than 18 years of age; 2) patients with missing serum creatinine date on admission; 3) dialysis patients; 4) patients with severe liver failure, severe brain disease, severe valvular heart disease, severe or decompensated heart failure, or terminal malignant tumor.

Serum creatinine (Scr) was measured on admission. Chronic kidney disease epidemiology collaboration (CKDEPI) Scr formula ${ }^{15}$ was used to calculate eGFR at admission. Patients were divided into three groups according to baseline renal function defined by admission eGFR values: Group A: eGFR $\geq 90 \mathrm{~mL} / \mathrm{min}$ (normal renal function); Group B: eGFR 60 to $<90 \mathrm{~mL} / \mathrm{min}$ (mild impaired renal function); Group $\mathrm{C}$ : eGFR $<60 \mathrm{~mL} / \mathrm{min}$ (moderate or severe renal dysfunction).

CKD-EPI Scr formula: GFR $=141 \times \min (\mathrm{Scr} / \kappa, 1)^{\alpha} \times \max$ $(\mathrm{Scr} / \kappa, 1)^{-1.209} \times 0.993^{\mathrm{Age}} \times 1.018$ [if female] $\times 1.159$ [if black] . Scr is serum creatinine; $\kappa$ is 0.7 for females and 0.9 for males; $\alpha$ is -0.329 for females and -0.411 for males; min indicates the minimum of Scr/ $\kappa$ or 1 ; and max indicates the maximum of Scr/ $\kappa$ or 1 .

\section{Endpoint and Follow-Up}

The endpoint was all-cause death during follow-up after discharge. Patients were followed up at outpatient clinics or using a telephone questionnaire at the date of discharge, then at 1, 6, and 12 months later, then annually after that. Follow-up methods included outpatient follow-up, rehospitalization, and telephone follow-up. Information about death was obtained from hospital records or via contact with the patients' guardians. Trained physicians collected the baseline and follow-up data.

\section{Statistical Analysis}

The SPSS software version 26.0 was used in the statistical analysis. Baseline demographics and clinical characteristics were compared among various eGFR levels. Categorical variables were presented as counts and percentages, and chi-square test was used for comparison among groups. Continuous variables of the normal distribution were presented as means \pm standard deviation, and One-way ANOVA was used for comparison among groups. Continuous variables of non-normal distribution were presented as median [interquartile range (IQR)], using Kruskal-Wallis $H$-test for comparisons among groups. The cumulative event rate was determined by Kaplan-Meier analysis with the Log rank test. A Cox proportional hazards model was used to determine independent predictors of the time-to-event (death), and to calculate hazard ratios (HRs) with $95 \%$ confidence intervals (CIs). By examining the relationships between the eGFR and death, we adjusted for variables that were 
different among eGFR levels (using p-value <0.1) and variables of known prognostic importance. These variables included age, gender, hypertension, diabetes, current smoking, prior history of coronary artery disease (CAD), prior history of chronic obstructive pulmonary disease (COPD), chest pain/distress, heart rate, systolic blood pressure (SBP), hemoglobin, STEMI vs NSTE-ACS, multiple coronary artery lesions, and percutaneous coronary intervention (PCI).

A two-sided p-value $<0.05$ was considered statistically significant in all analyses. GraphPad Prism version 8.0 was used to draw figures.

\section{Results}

\section{Clinical Characteristics}

A total of 1860 ACS patients were included in the study, among which the mean age was $65.6 \pm 13.9$ years and $71.2 \%(1342 / 1860)$ were male. Mild impaired renal function was present in 746 patients $(40.1 \%)$, whereas 400 patients $(21.5 \%)$ had moderate or severe dysfunction. During the follow of 15 months (10 months, 22 months), $261(14.0 \%)$ all-cause deaths were observed, of which 139 $(34.8 \%)$ occurred in patients with moderate or severe renal dysfunction, $94(12.6 \%)$ in mild impaired renal function, and $28(3.9 \%)$ normal renal function.

The baseline characteristics of the three groups are shown in Table 1.

Generally, patients with lower eGFR generally had a worse cardiovascular risk profile (Table 1). Compared to other groups, patients with moderate or severe renal dysfunction were more likely to be older, with a higher prevalence of hypertension, diabetes, CAD, COPD, stroke/ transient ischemic attack (TIA), cardiogenic shock, multivessel CAD, and calcified lesions ( $p$-value <0.05). However, smoking and chest pain/chest distress were less in this group ( $\mathrm{p}$-value $<0.05$ ). Notably, the proportion of patients with moderate or severe renal function undergoing PCI was lower than in the other two groups (p-value $<0.05)$. Nevertheless, there was no significant difference among the three groups regarding the history of myocardial infarction (MI), PCI, peripheral artery disease, or hospital admission. In-hospital mortality among patients with moderate or severe renal dysfunction was significantly higher than in the other two groups $(18.0 \%$ vs $5.8 \%$ vs $2.1 \%$, p-value $<0.001$ ).

The angiotensin converting enzyme inhibitor (ACEI)/ angiotensin receptor blocker (ARB) utilization rate in the moderate or severe renal dysfunction group was slightly lower ( $p$-value $<0.05$ ), but the utilization of diuretics was significantly higher than in the other two groups (p-value $<0.001)$. On the other hand, there was no significant difference in the utilization rate of antiplatelet agents, dual antiplatelet agents, statins, beta-blockers, and proton pump inhibitors (PPIs) among the three groups (p-value $>0.05$ ).

\section{Clinical Outcome}

The Kaplan-Meier survival curves showed that patients with a worse eGFR level had significantly higher rates of all-cause mortality (p-value $<0.001$, Figure 1). The allcause mortality rates for moderate or severe renal dysfunction group and mild impaired renal function were 38.9\% and $13.6 \%$, respectively, significantly higher than that in the normal renal function group $(5.6 \%)$ (p-value $<0.001)$.

In multivariable Cox regression hazard analysis in which eGFR was introduced as a categorical variable, age, SBP, heart rate, renal function levels, STEMI, and PCI were independently associated with all-cause death. On the other hand, when eGFR was introduced as a continuous variable, the independent predictors of allcause death were age, SBP, heart rate, eGFR, STEMI and PCI (Table 2).

In subgroup analysis limited to STEMI patients, the all-cause mortality rate in the moderate or severe renal dysfunction group was significantly higher than the other two groups $(39.5 \%$ vs $16.1 \%$ vs $5.0 \%$, moderate or severe renal dysfunction vs mild impaired renal function vs normal renal function, $\mathrm{p}$-value $<0.001)$. These results were consistent among patients with NSTE-ACS $(29.2 \%$ vs $8.6 \%$ vs $7.2 \%$, moderate or severe renal dysfunction vs mild impaired renal function vs normal renal function, p-value $<0.001$ ). As shown in the Kaplan-Meier survival curves in patients diagnosed with STEMI (Figure 2A), cumulative all-cause mortality during the follow-up of moderate or severe renal dysfunction group, mild renal insufficiency group, and normal renal function group decreased in turn ( $\mathrm{p}$-value $<0.001)$. In patients diagnosed with NSTE-ACS, the cumulative all-cause mortality during follow-up of moderate or severe renal dysfunction group was significantly higher than in the other two groups (p-value $<0.001$, Figure 2B). The Cox regression analysis showed that for STEMI patients, the independent predictors of all-cause death were age, SBP, heart rate, eGFR, and PCI. On the other hand, for NSTE-ACS patients, the independent predictors of all-cause death were age, SBP, heart rate, and eGFR (Table 3). 
Table I Baseline Characteristics

\begin{tabular}{|c|c|c|c|c|c|}
\hline \multirow[t]{2}{*}{ Characteristics } & \multicolumn{4}{|l|}{ eGFR } & \multirow[t]{2}{*}{ p value } \\
\hline & Total & $\begin{array}{l}\text { Normal } \\
(\geq 90 \mathrm{~mL} / \mathrm{min})\end{array}$ & $\begin{array}{l}\text { Mild (60 to } \\
<90 \mathrm{~mL} / \mathrm{min})\end{array}$ & $\begin{array}{l}\text { Moderate or Severe } \\
(<60 \mathrm{~mL} / \mathrm{min})\end{array}$ & \\
\hline No. of patients-No.\% & 1860 & $714(38.4)$ & $746(40.1)$ & $400(21.5)$ & \\
\hline Age (yrs) & $65.6 \pm 13.9$ & $55.1 \pm 11.4$ & $69.8 \pm 11.1$ & $76.1 \pm 10.1$ & $<0.001$ \\
\hline Sex (Male) -No.\% & $1342(7 \mid .2)$ & $566(79.3)$ & $534(71.6)$ & $242(60.5)$ & $<0.001$ \\
\hline Current smoker-No.\% & $717(38.5)$ & $35 I(49.3)$ & $262(35.5)$ & $104(26.2)$ & $<0.001$ \\
\hline \multicolumn{6}{|l|}{ Medical history-No.\% } \\
\hline History of hypertension & $1056(56.8)$ & $329(46.1)$ & $447(59.9)$ & $280(70.0)$ & $<0.001$ \\
\hline History of diabetes & $483(26.0)$ & $152(21.3)$ & $195(26.1)$ & $136(34.0)$ & $<0.001$ \\
\hline History of CAD & $312(16.8)$ & $95(13.4)$ & $|4|(\mid 9.0)$ & $76(19.0)$ & 0.007 \\
\hline History of stroke or TIA & $90(4.8)$ & $14(2.0)$ & $42(5.7)$ & $34(8.5)$ & $<0.001$ \\
\hline History of COPD & $78(4.2)$ & $8(I .1)$ & $43(5.8)$ & $27(6.8)$ & $<0.001$ \\
\hline History of peripheral arterial diseases & $16(0.9)$ & $3(0.4)$ & $7(0.9)$ & $6(1.5)$ & 0.169 \\
\hline Previous MI & $97(5.2)$ & $30(4.2)$ & $43(5.8)$ & $24(6.0)$ & 0.311 \\
\hline Previous $\mathrm{PCl}$ & $114(6.1)$ & $36(5.1)$ & $49(6.6)$ & $29(7.3)$ & 0.282 \\
\hline SBP $(\mathrm{mmHg})$ & $133 \pm 26$ & $133 \pm 22$ & $134 \pm 25$ & $|29 \pm 3|$ & 0.019 \\
\hline Heart rate (beats per min) & $77(67,90)$ & $76(68,89)$ & $75(65,88)$ & $80(68,94)$ & $<0.001$ \\
\hline Cardiogenic shock-No.\% & $134(7.2)$ & $21(3.0)$ & $48(6.5)$ & $65(16.4)$ & $<0.001$ \\
\hline Admission by ambulance-No.\% & $146(7.8)$ & $50(7.0)$ & $53(7.1)$ & $43(10.8)$ & 0.052 \\
\hline Chest pain/ chest distress-No.\% & $1688(90.7)$ & $674(97.0)$ & 676(93.9) & $338(87.8)$ & $<0.001$ \\
\hline \multicolumn{6}{|l|}{ Diagnosis-No.\% } \\
\hline STEMI & $992(53.3)$ & $379(53.1)$ & $398(53.4)$ & $215(53.8)$ & $<0.001$ \\
\hline $\mathrm{Hb}(\mathrm{g} / \mathrm{L})$ & $|33 \pm 2|$ & $|4| \pm 18$ & $132 \pm 19$ & $120 \pm 23$ & $<0.001$ \\
\hline \multicolumn{6}{|l|}{ Coronary artery-No.\% } \\
\hline Multi-vessel diseases & $848(45.6)$ & $299(41.9)$ & $376(50.4)$ & $173(43.3)$ & 0.003 \\
\hline Calcified lesions & $105(5.6)$ & $18(2.6)$ & $54(7.7)$ & $26(7.9)$ & $<0.001$ \\
\hline $\mathrm{PCl}-\mathrm{No} . \%$ & $1100(59.1)$ & $440(61.6)$ & $47 I(63 . I)$ & $189(47.3)$ & $<0.001$ \\
\hline \multicolumn{6}{|l|}{ Social benefit } \\
\hline Hospital stay (days) & $8(6,11)$ & $8(6,10)$ & $9(7,12)$ & $9(5,13)$ & $<0.001$ \\
\hline Hospitalization cost (ten thousand yuan) & $3.21(1.05,4.47)$ & $3.20(1.04,4.25)$ & $3.36(1.09,4.69)$ & $2.74(1.00,4.56)$ & 0.060 \\
\hline \multicolumn{6}{|l|}{ Medications at discharge-No.\% } \\
\hline Antiplatelet agents & $1558(90.1)$ & $63 I(90.3)$ & $633(90.0)$ & 294(89.6) & 0.950 \\
\hline Dual antiplatelet agents & $1332(77.0)$ & $543(77.7)$ & $547(77.8)$ & $242(73.8)$ & 0.264 \\
\hline Statins & $1540(89.0)$ & $624(89.3)$ & $625(88.9)$ & $291(88.7)$ & 0.397 \\
\hline ACEI/ARB & $896(51.8)$ & $349(49.9)$ & $389(55.3)$ & $158(48.2)$ & 0.021 \\
\hline Beta-blockers & $1101(63.6)$ & $462(66.1)$ & $443(63.0)$ & 196(59.8) & 0.061 \\
\hline PPls & $566(32.7)$ & $226(32.3)$ & $225(32.0)$ & $115(35.1)$ & 0.701 \\
\hline Diuretics & $310(17.9)$ & 69(9.9) & $128(\mid 8.2)$ & $113(34.5)$ & $<0.001$ \\
\hline In-hospital mortality & $130(7.0)$ & $15(2.1)$ & $43(5.8)$ & $72(18.0)$ & $<0.001$ \\
\hline
\end{tabular}

Abbreviations: eGFR, estimated glomerular filtration rate; CAD, coronary artery disease; TIA, transient ischemic attack; COPD, chronic obstructive pulmonary disease; $\mathrm{MI}$, myocardial infarction; PCl, percutaneous coronary intervention; SBP, systolic blood pressure; STEMI, ST-segment elevation myocardial infarction; Hb, hemoglobin; PCl, percutaneous coronary intervention; ACEl, angiotensin converting enzyme inhibitor; ARB, angiotensin receptor blocker; PPIs, proton pump inhibitors. 


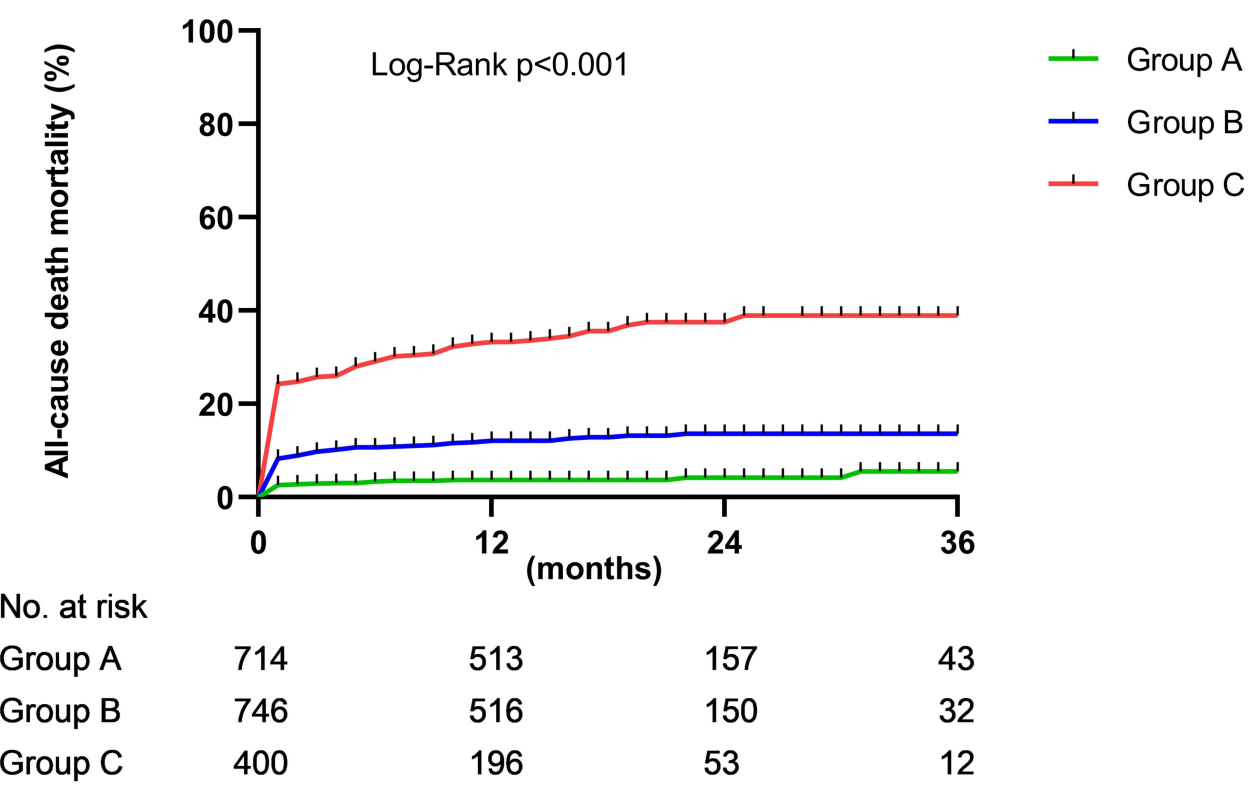

Figure I Kaplan-Meier estimates of the rates of all-cause. Group A: ACS patients with normal renal function; Group B: ACS patients with mild impaired renal function; Group C: ACS patients with moderate or severe renal dysfunction. 'Represented censored data.

\section{Discussion}

This study demonstrated that renal insufficiency is an independent risk factor for all-cause mortality in Chinese patients with ACS. The magnitude of risk was proportionate to the decline in renal function. The cumulative allcause mortality in both moderate or severe renal dysfunction group and mild renal dysfunction group was significantly higher than those patients with normal kidney function (p-value $<0.001$ ). In addition, the proportion of ACS with renal insufficiency was $61.6 \%(21.5 \%$ for moderate or severe renal dysfunction and $40.1 \%$ for mild impaired renal function). For such patients, we should

Table 2 Multivariable Cox Proportional Hazard Analyses for All-Cause Death in ACS Patients with Renal Insufficiency

\begin{tabular}{|c|c|c|c|c|c|c|}
\hline & \multicolumn{3}{|c|}{ eGFR as Categorical Variable } & \multicolumn{3}{|c|}{ eGFR as Continuous Variable } \\
\hline & HR & $95 \% \mathrm{Cl}$ & p value & HR & $95 \% \mathrm{Cl}$ & p value \\
\hline Male & 1.13 & $0.84-1.52$ & 0.419 & 1.14 & $0.85-1.52$ & 0.400 \\
\hline Age & 1.05 & $1.03-1.06$ & $<0.001$ & 1.05 & $1.03-1.06$ & $<0.001$ \\
\hline Smoking & 1.06 & $0.77-1.46$ & 0.728 & 1.07 & $0.78-1.47$ & 0.669 \\
\hline Hypertension & 1.09 & $0.82-1.44$ & 0.565 & 1.04 & $0.78-1.38$ & 0.791 \\
\hline Diabetes & 1.10 & $0.83-1.46$ & 0.515 & 1.06 & $0.80-1.40$ & 0.702 \\
\hline Prior CAD & 1.12 & $0.80-1.56$ & 0.525 & 1.14 & $0.82-1.60$ & 0.433 \\
\hline Prior COPD & 0.98 & $0.59-1.62$ & 0.928 & 0.98 & $0.59-1.62$ & 0.933 \\
\hline Chest pain/ chest distress & 0.71 & $0.48-1.03$ & 0.070 & 0.79 & $0.54-1.15$ & 0.219 \\
\hline Heart rate & 1.01 & $1.01-1.02$ & $<0.001$ & 1.01 & $1.01-1.02$ & $<0.001$ \\
\hline SBP & 0.99 & $0.98-0.99$ & $<0.00$ I & 0.99 & $0.98-0.99$ & $<0.001$ \\
\hline $\mathrm{Hb}$ & 1.00 & $0.99-1.00$ & 0.496 & 1.00 & $0.99-1.01$ & 0.939 \\
\hline eGFR & & & $<0.001$ & 0.98 & $0.98-0.99$ & $<0.001$ \\
\hline eGFR $\geq 90$ & - & - & - & & & \\
\hline $60 \leq e G F R<90$ & 1.57 & $0.96-2.57$ & 0.071 & & & \\
\hline eGFR $<60$ & 2.72 & I.62-4.57 & $<0.001$ & & & \\
\hline STEMI & 1.73 & I.29-2.34 & $<0.001$ & 1.71 & $1.26-2.30$ & $<0.001$ \\
\hline Multi-vessel diseases & 1.10 & $0.81-1.49$ & 0.564 & 1.34 & $0.83-1.55$ & 0.423 \\
\hline $\mathrm{PCl}$ & 0.52 & $0.37-0.72$ & $<0.001$ & 0.52 & $0.37-0.72$ & $<0.001$ \\
\hline
\end{tabular}

Abbreviations: eGFR, estimated glomerular filtration rate; CAD, coronary artery disease; COPD, chronic obstructive pulmonary disease; SBP, systolic blood pressure; Hb, hemoglobin; STEMI, ST-segment elevation myocardial infarction; $\mathrm{PCI}$, percutaneous coronary intervention. 


\section{A}
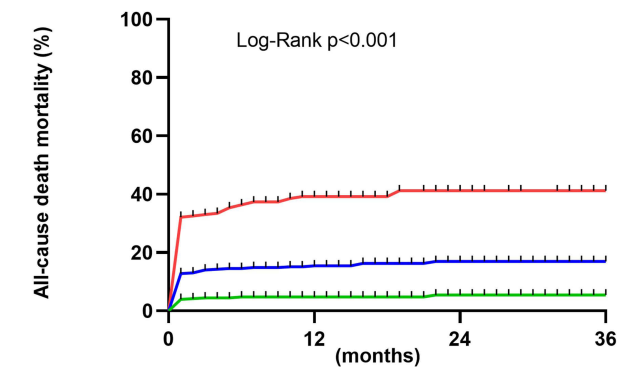

$$
\begin{array}{r}
\perp \text { Group A } \\
+ \text { Group B } \\
+ \text { Group C }
\end{array}
$$

No. at risk

Group A

Group B

Group C

$$
\begin{aligned}
& 379 \\
& 398
\end{aligned}
$$

215

\begin{abstract}
270
261
\end{abstract}

94

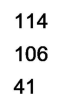

41
B
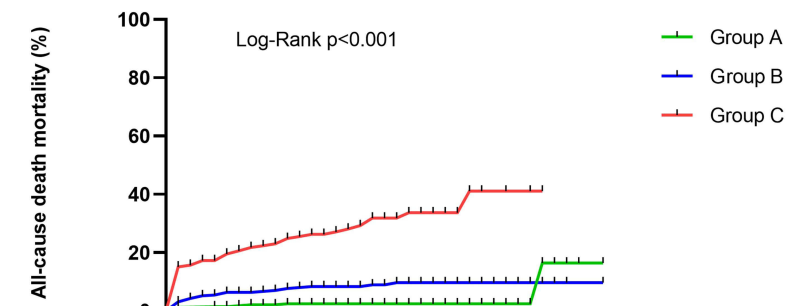

No. at risk

Group A

Group B Group C

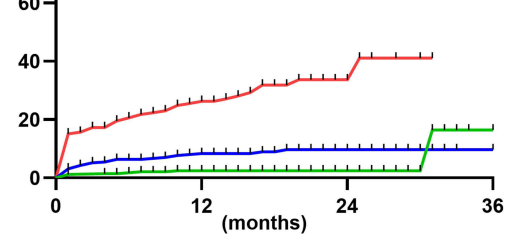

$\begin{array}{llll}335 & 243 & 43 & 2 \\ 348 & 255 & 44 & 2 \\ 185 & 102 & 12 & \end{array}$

Figure 2 (A) The cumulative rate of all-cause death in STEMI patients with renal insufficiency. (B) The cumulative rate of all-cause death in NSTE-ACS patients with renal insufficiency. 'Represented censored data.

take active treatment and perioperative management to improve their prognosis.

In the past decades, the prognosis of ACS patients has improved significantly; however, the prognosis of ACS patients with renal insufficiency is still poor. Data from the Global Registry of Acute Coronary Events registry showed that in-hospital mortality of STEMI patients with eGFR $<30 \mathrm{~mL} / \mathrm{min} / 1.73 \mathrm{~m}^{2}$ was up to $29.2 \%{ }^{16}$ In another large study, the Harmonizing Outcomes With Revascularization and Stents in Acute Myocardial Infarction trial, the 1-year all-cause mortality of STEMI patients with GFR $<30 \mathrm{~mL} / \mathrm{min} / 1.73 \mathrm{~m}^{2}$ was $36.5 \% .{ }^{17} \mathrm{We}$ found that during the follow-up period, all-cause mortality rate in the moderate or severe renal dysfunction group was $38.9 \%$, which was significantly higher than the normal renal function group (5.6\%). Moreover, the all-cause mortality rate in the mild renal dysfunction group (13.6\%) was also higher than the normal renal function group. ACS patients with mild renal insufficiency have a relatively poor long-term prognosis; however, they have not received enough attention.

There are several possible mechanisms for poor prognosis in ACS patients with renal insufficiency. Patients with

\begin{tabular}{|c|c|c|c|c|c|c|}
\hline & \multicolumn{3}{|c|}{ STEMI } & \multicolumn{3}{|c|}{ NSTE-ACS } \\
\hline & HR & $95 \% \mathrm{Cl}$ & p value & HR & $95 \% \mathrm{Cl}$ & $p$ value \\
\hline Male & 1.45 & $0.99-2.11$ & 0.056 & 0.77 & $0.47-1.25$ & $0.28 \mathrm{I}$ \\
\hline Age & 1.05 & $1.03-1.06$ & $<0.001$ & 1.05 & $1.03-1.08$ & $<0.001$ \\
\hline Smoking & 1.17 & $0.78-1.75$ & 0.461 & 0.89 & $0.52-1.52$ & 0.667 \\
\hline Hypertension & 1.19 & $0.84-1.68$ & 0.337 & 0.78 & $0.48-1.27$ & 0.324 \\
\hline Diabetes & 1.13 & $0.79-1.63$ & 0.504 & 0.91 & $0.57-1.45$ & 0.681 \\
\hline Prior CAD & 1.31 & $0.8 \mathrm{I}-2.10$ & 0.270 & 0.93 & $0.58-1.50$ & 0.774 \\
\hline Prior COPD & 0.99 & $0.48-2.06$ & 0.979 & 0.94 & $0.46-1.92$ & 0.870 \\
\hline Chest pain/ chest distress & 0.78 & $0.47-1.30$ & 0.339 & 0.85 & $0.46-1.55$ & 0.588 \\
\hline Heart rate & 1.01 & $1.00-1.02$ & 0.006 & 1.01 & $1.00-1.02$ & 0.005 \\
\hline SBP & 0.99 & $0.98-1.00$ & 0.001 & 0.99 & $0.98-0.99$ & 0.001 \\
\hline $\mathrm{Hb}$ & 1.00 & $0.99-1.01$ & 0.943 & 1.00 & $0.99-1.01$ & 0.814 \\
\hline eGFR & 0.99 & $0.98-1.00$ & 0.004 & 0.98 & $0.97-0.99$ & $<0.001$ \\
\hline Multi-vessel diseases & 1.14 & $0.78-1.67$ & 0.487 & 1.04 & $0.59-1.84$ & 0.892 \\
\hline $\mathrm{PCl}$ & 0.44 & $0.30-0.65$ & $<0.001$ & 0.76 & $0.42-1.36$ & 0.350 \\
\hline
\end{tabular}
reduced eGFR are often associated with various cardiovascular risk factors, including elderly, hypertension, diabetes, dyslipidemia, hyperhomocysteinemia and oxidative stress.

Table 3 Multivariable Cox Proportional Hazard Analyses for All-Cause Death in STEMI Patients and NSTEMI Patients

Abbreviations: STEMI, ST-segment elevation myocardial infarction; NSTE-ACS, non-ST-segment elevation acute coronary syndrome; CAD, coronary artery disease; COPD, chronic obstructive pulmonary disease; SBP, systolic blood pressure; Hb, hemoglobin; eGFR, estimated glomerular filtration rate; PCl, percutaneous coronary intervention. 
These risk factors could accelerate the process of atherosclerosis and increase the risk of thrombosis. ${ }^{18}$ Also, patients with lower eGFR often have anemia and erythropoietin resistance, malnutrition, abnormal calcium and phosphorus metabolism, sodium and volume overload, endothelial dysfunction associated with urotoxin, LDL oxidation, and smooth muscle cell proliferation induced by oxidative stress. ${ }^{19,20}$ These adverse factors interact with each other gradually to form a vicious circle, which can accelerate coronary atherosclerosis, cardiac remodeling with left ventricular hypertrophy, and further lead to decreased coronary perfusion and left ventricular systolic and diastolic dysfunction. ${ }^{21}$ Furthermore, the decline in eGFR is closely related to the systemic inflammatory state. ${ }^{22}$ The increase in inflammatory factors in patients with renal insufficiency could lead to an increased risk of CVD event. ${ }^{23}$ Some studies have also indicated that ACS patients with CKD are less likely to receive evidence-based treatment, including early interventional therapy. ${ }^{24,25}$ This could be complicated by the fact that ACS symptoms in patients with chronic kidney disease may be atypical, delaying diagnosis and leading to adverse outcomes. ${ }^{26}$

On the other hand, the poor prognosis of ACS patients with renal insufficiency may be due to the proportion of patients with moderate or severe renal dysfunction receiving PCI was low, while the proportion of STEMI was not significantly different from the other two groups. This finding is consistent with previous studies ${ }^{25,27}$ Large randomized clinical trials cited in ACS guidelines often exclude patients with renal insufficiency, resulting in insufficient evidence. In addition, compared with ACS patients with normal renal function, ACS patients with renal insufficiency have more serious, complex, and calcified coronary artery lesions, as well as more multivessel lesions. ${ }^{28}$ Thus, the operation is more difficult in this portion of patients, but with higher risk and lower success rate. Simultaneously, patients with renal insufficiency are more prone to hemorrhage, contrast-induced nephropathy and aggravation of renal failure after PCI treatment. $^{29,30}$ A higher rate of stent thrombosis also results in a low rate of PCI in patients with renal insufficiency. ${ }^{31} \mathrm{We}$ also found that the utilization rate of taking guideline-based drugs for discharged ACS patients, especially for dual antiplatelet agents and ACEI/ARB, was relatively low. This reflected a matter of fact that less frequent utilization of guideline-based cardiovascular medications between 2014 and 2019, consistent with previous studies. ${ }^{32,33}$ Concern about an increased incidence of adverse drug reactions in patients with renal insufficiency may also result in the less frequent utilization. However, a series of clinical studies have proved that ACS patients with renal insufficiency can also benefit from evidence-based medicine and invasive treatment. ${ }^{18,34}$ Therefore, current guidelines recommended the same treatment for these patients as for patients with normal renal function, except from minor dose adjustments. ${ }^{35-37}$ ACS high-risk patients should actively follow the guidelines for coronary angiography, STEMI patients should follow the guidelines for reperfusion therapy. Regarding this portion of patients, physicians may adopt a more active treatment strategy.

Our study has some strengths. Although the effect of renal function on the prognosis of ACS patients has been well documented, the patients enrolled were mainly with moderate or severe renal dysfunction, or with CKD. Limited studies focus on the patients with mild renal dysfunction. Furthermore, similar studies were seldom conducted in Asian populations or with just a short followup period. ${ }^{38}$ Our study was a multicenter study with ACS patients enrolled from 11 hospitals in Chengdu, who were followed for a long time. Noteworthy, in our analysis, we used the CKD-EPI formula proposed by the Chronic Kidney Disease Epidemiology Collaboration. CKD-EPI has a higher accuracy than Cockcroft-Gault formula and MDRD formula, and it can better predict the risk of CVD and cerebrovascular diseases.

Our study has several limitations. First, this is a retrospective study, where the sample size is relatively small, which may lead to data bias. Second, previous serum creatinine levels were not available to us, and therefore some patients may have presented with acute rather than chronic kidney disease. In addition, most patients only measured serum creatinine on admission, and therefore we do not know the dynamic changes of serum creatinine level in patients. However, in real-world studies, it is difficult to measure each patient in a large sample more than once during hospitalization. Furthermore, some patients were followed up for a short time because the time of admission was inconsistent.

\section{Conclusion}

In this study, among Chinese patients, the long-term prognosis of ACS patients with renal insufficiency was unfavorable, and the mortality was relatively high. A higher decline in renal function was associated with a worse prognosis among ACS patients. Age, SBP, heart rate, 
eGFR (as a categorical variable or a continuous variable), STEMI, and PCI were independent predictors of all-cause death during follow-up. The proportion of ACS patients with moderate or severe renal dysfunction receiving PCI treatment or using dual antiplatelets and ACEI/ARB was low, and in the real world, these patients are not fully treated with evidence-based medicine. Attention to the standardized treatment recommended by the guidelines is warranted.

\section{Data Sharing Statement}

The datasets used are available from the corresponding authors on reasonable requests.

\section{Ethics Approval and Consent to Participate}

The study was conducted according to the guidelines of the Declaration of Helsinki. The study was approved by the ethics committee of The Third People's Hospital of Chengdu. This study was an observational study and was a collection and research of previously archived data and documents, which were public resources. Through the data collected in this study, the subjects could not be sought out, and the research project did not involve personal privacy and commercial interests, which complied with the regulations of "Measures for ethical review of biomedical research involving human beings" issued by China in 2016 and the Declaration of Helsinki, therefore the Ethics Committee of the Third People's Hospital of Chengdu approved the waiver for informed consent. Statement of patient data confidentiality: After completion of the data collection in our study, the personal information of identifiable subjects related to the data was deleted, and anonymous numbers were used to replace it. The data from our study were holistic rather than individual. In addition, the data of this study were collected and managed by trained professionals to avoid data leakage.

\section{Acknowledgments}

This study was supported by the Science and Technology Department of Sichuan, China [Grant number 2020 YJ0483 and 2021YJ0215]. The authors would like to express their gratitude to EditSprings (https://www.edit springs.com/) for the expert linguistic services provided.

\section{Disclosure}

All authors declare that there is no conflict of interest.

\section{References}

1. Piepoli MF, Hoes AW, Brotons C, Hobbs RFD, Corra U. Task Force for the 2016 guidelines on cardiovascular disease prevention in clinical practice. Main messages for primary care from the 2016 European Guidelines on cardiovascular disease prevention in clinical practice. Eur J Gen Pract. 2018;24(1):51-56. doi:10.1080/ 13814788.2017.1398320

2. Virani SS, Alonso A, Aparicio HJ, et al. Heart disease and stroke statistics-2021 update: a report from the American Heart Association. Circulation. 2021;143(8):e254-e743. doi:10.1161/CIR.000000000 0000950

3. Petersen JK, Butt JH, Yafasova A, et al. Incidence of ischaemic stroke and mortality in patients with acute coronary syndrome and first-time detected atrial fibrillation: a nationwide study. Eur Heart $J$. 2021:ehab575. doi:10.1093/eurheartj/ehab575

4. Committee of the Report on Cardiovascular Health and Diseases in China. Report on cardiovascular health and diseases burden in China: an updated summary of 2020. Chin Circ J. 2021;36(6):521-545.

5. Bikbov B, Purcell CA, Levey AS; GBD Chronic Kidney Disease Collaboration. Global, regional, and national burden of chronic kidney disease, 1990-2017: a systematic analysis for the Global Burden of Disease Study 2017. Lancet. 2020;395(10225):709-733. doi:10.1016/S0140-6736(20)30045-3

6. Zhang L, Wang F, Wang L, et al. Prevalence of chronic kidney disease in China: a cross-sectional survey. Lancet. 2012;379 (9818):815-822. doi:10.1016/S0140-6736(12)60033-6

7. Pilmore HL, Xiong F, Choi Y, et al. Impact of chronic kidney disease on mortality and cardiovascular outcomes after acute coronary syndrome: a nationwide data linkage study (ANZACS-QI 44). Nephrology. 2020;25(7):535-543. doi:10.1111/nep.13703

8. Rott D, Klempfner R, Goldenberg I, Matetzky S, Elis A. Temporal trends in the outcomes of patients with acute myocardial infarction associated with renal dysfunction over the past decade. Eur J Intern Med. 2016;29:88-92. doi:10.1016/j.ejim.2015.12.020

9. Dohi T, Kasai T, Miyauchi K, et al. Prognostic impact of chronic kidney disease on 10-year clinical outcomes among patients with acute coronary syndrome. $J$ Cardiol. 2012;60(6):438-442. doi:10.1016/j.jjcc.2012.08.007

10. Melloni C, Cornel JH, Hafley G, et al. Impact of chronic kidney disease on long-term ischemic and bleeding outcomes in medically managed patients with acute coronary syndromes: insights from the TRILOGY ACS Trial. Eur Heart J Acute Cardiovasc Care. 2016;5 (6):443-454. doi:10.1177/2048872615598631

11. Sarnak MJ, Amann K, Bangalore S, et al. Chronic kidney disease and coronary artery disease: JACC state-of-the-art review. J Am Coll Cardiol. 2019;74(14):1823-1838. doi:10.1016/j.jacc.2019.08.1017

12. Foley RN, Parfrey PS, Sarnak MJ. Epidemiology of cardiovascular disease in chronic renal disease. J Am Soc Nephrol. 1998;9(12 Suppl):S16-23.

13. Collet JP, Thiele H, Barbato E, et al. 2020 ESC guidelines for the management of acute coronary syndromes in patients presenting without persistent ST-segment elevation. Eur Heart J. 2021;42 (14):1289-1367. doi:10.1093/eurheartj/ehaa575

14. Thygesen K, Alpert JS, Jaffe AS, et al. Third universal definition of myocardial infarction. Eur Heart J. 2012;33(20):2551-2567. doi:10.1093/eurheartj/ehs184

15. Levey AS, Stevens LA, Schmid $\mathrm{CH}$, et al. A new equation to estimate glomerular filtration rate. Ann Intern Med. 2009;150 (9):604-612. doi:10.7326/0003-4819-150-9-200905050-00006

16. Medi C, Montalescot G, Budaj A, et al. Reperfusion in patients with renal dysfunction after presentation with ST-segment elevation or left bundle branch block: GRACE (Global Registry of Acute Coronary Events). JACC Cardiovasc Interv. 2009;2(1):26-33. doi:10.1016/j. jcin.2008.09.010 
17. Saltzman AJ, Stone GW, Claessen BE, et al. Long-term impact of chronic kidney disease in patients with ST-segment elevation myocardial infarction treated with primary percutaneous coronary intervention: the HORIZONS-AMI (Harmonizing outcomes with revascularization and stents in acute myocardial infarction) trial. JACC Cardiovasc Interv. 2011;4(9):1011-1019. doi:10.1016/j.jcin.2011.06.012

18. Sarnak MJ, Levey AS, Schoolwerth AC, et al. Kidney disease as a risk factor for development of cardiovascular disease: a statement from the American heart association councils on kidney in cardiovascular disease, high blood pressure research, clinical cardiology, and epidemiology and prevention. Hypertension. 2003;42 (5):1050-1065. doi:10.1161/01.HYP.0000102971.85504.7c

19. McCullough Peter A. Cardiorenal risk: an important clinical intersection. Rev Cardiovasc Med. 2002;3(2):71-76.

20. Cai Q, Mukku VK, Ahmad M. Coronary artery disease in patients with chronic kidney disease: a clinical update. Curr Cardiol Rev. 2013;9(4):331-339. doi:10.2174/1573403X10666140214122234

21. Ronco C, Haapio M, House AA, Anavekar N, Bellomo R. Cardiorenal syndrome. J Am Coll Cardiol. 2008;52(19):1527-1539. doi:10.1016/j.jacc.2008.07.051

22. Papayianni A, Alexopoulos E, Giamalis P, et al. Circulating levels of ICAM-1, VCAM-1, and MCP-1 are increased in haemodialysis patients: association with inflammation, dyslipidaemia, and vascular events. Nephrol Dial Transplant. 2002;17(3):435-441. doi:10.1093/ndt/17.3.435

23. Baigent $\mathrm{C}$, Landray MJ, Reith $\mathrm{C}$, et al. The effects of lowering LDL cholesterol with simvastatin plus ezetimibe in patients with chronic kidney disease (Study of heart and renal protection): a randomised placebo-controlled trial. Lancet. 2011;377(9784):2181-2192. doi:10.1016/S0140-6736(11)60739-3

24. Chertow GM, Normand SL, McNeil BJ. "Renalism": inappropriately low rates of coronary angiography in elderly individuals with renal insufficiency. $J$ Am Soc Nephrol. 2004;15(9):2462-2468. doi:10.1097/01.ASN.0000135969.33773.0B

25. Wright RS, Reeder GS, Herzog CA, et al. Acute myocardial infarction and renal dysfunction: a high-risk combination. Ann Intern Med. 2002;137(7):563-570. doi:10.7326/0003-4819-137-7-200210010-00007

26. Levin A, Tonelli M, Bonventre J, et al. Global kidney health 2017 and beyond: a roadmap for closing gaps in care, research, and policy. Lancet. 2017;390(10105):1888-1917. doi:10.1016/S0140-6736(17)30788-2

27. Bagai A, Lu D, Lucas J, et al. Temporal trends in utilization of cardiac therapies and outcomes for myocardial infarction by degree of chronic kidney disease: a report from the NCDR chest pain-MI registry. $\mathrm{J} \mathrm{Am}$ Heart Assoc. 2018;7(24):e010394. doi:10.1161/JAHA.118.010394

28. Widimsky P, Rychlik I. Renal disease and acute coronary syndrome. Heart. 2010;96(1):86-92. doi:10.1136/hrt.2008.161034

29. Marenzi G, Lauri G, Assanelli E, et al. Contrast-induced nephropathy in patients undergoing primary angioplasty for acute myocardial infarction. J Am Coll Cardiol. 2004;44(9):1780-1785. doi:10.1016/ j.jacc. 2004.07 .043
30. Kowalczyk J, Lenarczyk R, Kowalski O, et al. Different types of renal dysfunction in patients with acute myocardial infarction treated with percutaneous coronary intervention. J Interv Cardiol. 2007;20 (2):143-152. doi:10.1111/j.1540-8183.2007.00253.x

31. Margolis G, Vig S, Flint N, et al. Prognostic implications of chronic kidney disease on patients presenting with ST-segment elevation myocardial infarction with versus without stent thrombosis. Cardiorenal Med. 2017;7(2):150-157. doi:10.1159/000455905

32. Du X, Patel A, Anderson CS, et al. Epidemiology of cardiovascular disease in china and opportunities for improvement: JACC international. $J$ Am Coll Cardiol. 2019;73(24):3135-3147. doi:10.1016/j.jacc.2019.04.036

33. Atkins ER, Du X, Wu Y, et al. Use of cardiovascular prevention treatments after acute coronary syndrome in China and associated factors. Int J Cardiol. 2017;241:444 449. doi:10.1016/j.ijcard.2017.03.057

34. Szummer K, Lundman P, Jacobson SH, et al. Influence of renal function on the effects of early revascularization in non-ST-elevation myocardial infarction: data from the Swedish Web-System for Enhancement and Development of Evidence-based care in Heart Disease Evaluated According to Recommended Therapies (SWEDEHEART). Circulation. 2009;120(10):851-858. doi:10.1161/CIRCULATIONAHA.108.838169

35. Smith SC Jr, Benjamin EJ, Bonow RO, et al. AHA/ACCF secondary prevention and risk reduction therapy for patients with coronary and other atherosclerotic vascular disease: 2011 update: a guideline from the American Heart Association and American College of Cardiology Foundation endorsed by the World Heart Federation and the Preventive Cardiovascular Nurses Association. J Am Coll Cardiol. 2011;58(23):2432-2446. doi:10.1016/j.jacc.2011.10.824

36. Piepoli MF, Hoes AW, Agewall S, et al. 2016 European guidelines on cardiovascular disease prevention in clinical practice: the Sixth Joint Task Force of the European Society of Cardiology and other societies on cardiovascular disease prevention in clinical practice (constituted by representatives of 10 societies and by invited experts) developed with the special contribution of the European Association for Cardiovascular Prevention \& Rehabilitation (EACPR). Eur Heart J. 2016;37(29):2315-2381. doi:10.1093/eurheartj/ehw106

37. Cheung AK, Chang TI, Cushman WC, et al. Executive summary of the KDIGO 2021 Clinical practice guideline for the management of blood pressure in chronic kidney disease. Kidney Int. 2021;99 (3):559-569. doi:10.1016/j.kint.2020.10.026

38. Peng Y, Du X, Li X, et al. Association of renal insufficiency with treatments and outcomes in patients with acute coronary syndrome in China. Int J Cardiol. 2021;323:7-12. doi:10.1016/j.ijcard.2020.08.022
International Journal of General Medicine

\section{Publish your work in this journal}

The International Journal of General Medicine is an international, peer-reviewed open-access journal that focuses on general and internal medicine, pathogenesis, epidemiology, diagnosis, monitoring and treatment protocols. The journal is characterized by the rapid reporting of reviews, original research and clinical studies

\section{Dovepress}

across all disease areas. The manuscript management system is completely online and includes a very quick and fair peer-review system, which is all easy to use. Visit http://www.dovepress.com/ testimonials.php to read real quotes from published authors. 